\title{
La confianza, la satisfacción, las normas relacionales, el oportunismo y la dependencia como antecedentes del compromiso organizacional del trabajador
}

Fecha de recepción: 14.03.2011

Fecha de aceptación: 23.05.2011

Sonia San Martín

Gutiérrez.

Departamento de Economía y Administración de Empresas, Universidad de Burgos

sanmargu@ubu.es

\section{Resumen}

El objetivo de este trabajo es conocer los determinantes más importantes del compromiso afectivo, normativo y de continuidad del trabajador con la empresa. Nuestra propuesta contempla tanto antecedentes de tipo relacional o interpersonal (confianza, satisfacción y normas relacionales) como de tipo económico (oportunismo empresarial y dependencia). El contraste de las hipótesis propuestas, con una muestra de trabajadores mexicanos, indica que tanto el compromiso afectivo como el normativo inciden en el de continuidad; la satisfacción, las normas relacionales y la percepción de oportunismo son los principales antecedentes del compromiso afectivo; y la confianza y la dependencia lo son del compromiso normativo. Por otra parte, la confianza y las normas relacionales contribuyen al aumento de la satisfacción laboral, mientras que los antecedentes del compromiso afectivo lo hacen también, indirectamente, en el compromiso normativo. Estos resultados arrojan interesantes implicaciones para el ámbito académico y profesional.

Palabras clave: relación laboral, compromiso organizacional, antecedentes relacionales o interpersonales, antecedentes económicos. 


\title{
Trust, satisfaction, relational norms, opportunism and dependence as antece- dents of employee organizational commitment
}

\begin{abstract}
The objective of this study is to research the most important antecedents of the affective, normative and continuance of employee's commitment in his/her relation with the firm. The proposal includes both relational or interpersonal antecedents (trust, satisfaction and relational norms) and economic antecedents (dependence and firm opportunism). The testing of the proposed hypotheses with a sample of Mexican employees shows that both affective and normative commitment influence continuance commitment; satisfaction, relational norms and perception of opportunism are the main antecedents of affective commitment; and trust and dependence are the main antecedents of normative commitment. Besides, trust and relational norms contribute to enhance labor satisfaction as well as the antecedents of affective commitment are also, indirectly, antecedents of normative commitment. These results show interesting implications for both the academic and the professional world.
\end{abstract}

Keywords: labor relation, organizational commitment, relational or interpersonal antecedents, economic antecedents.

\section{Introducción}

Es habitual tratar la relación laboral entre el empleado y la organización en términos del contrato psicológico que refleja los compromisos adquiridos, escritos o no, entre ambas partes (Robinson y Rousseau, 1994; seguido posteriormente por McDonald y Makin, 2000; y Winter y Jackson 2006). No obstante, hay otros enfoques (Guest, 1998) que enfatizan el estudio del estado de la relación laboral entendida también como un contrato psicológico en referencia con el cumplimiento de promesas y obligaciones y a su relación con las actitudes y el comportamiento en el trabajo. En esta línea hay varios investigadores que han estudiado cómo conseguir y mantener el compromiso organizacional (CO) de los trabajadores (Meyer y Allen, 1997; Bayona, Goñi y Madorran, 2000; Winter y Jackson, 2006), lo que es sumamente relevante y que contribuye a mejorar el desarrollo profesional de los empleados (Madero, 2010) y a mejorar los beneficios y eficiencia de la empresa. El objetivo principal de este trabajo es profundizar en el conocimiento de los factores conducentes al $\mathrm{CO}$ del trabajador con la empresa, distinguiendo los antecedentes del compromiso afectivo (CA), normativo (CN) y de continuidad (CC). 
Tras la revisión de la literatura que se realizó sobre relaciones laborales, las principales contribuciones de este trabajo son: a) es un estudio en el que se consideran antecedentes del $\mathrm{CO}$ de diferente naturaleza (relacional o interpersonal y económica) y en el que se aplican diferentes teorías para el desarrollo del modelo e hipótesis; b) es un trabajo que trata los antecedentes del CA, CN y CC, frente a la mayor parte de los investigadores que han tratado más los antecedentes del CA que los antecedentes del $\mathrm{CN}$; c) es un estudio de los antecedentes del CO para el caso mexicano y con un comentario de implicaciones para ese caso (precisamente, Elahee y Brooks (2004) resaltaron la conveniencia de indagar más en las diferencias entre comportamientos empresariales en México en relación con otros países); d) es una investigación que estima un modelo causal mediante la metodología de ecuaciones estructurales, mientras que la mayoría de estudios sobre OC son teóricos o no prueban causalidad entre las variables multi-ítem, como resaltan Robinson (1996) o McDonald y Makin (2000).

\section{El compromiso organizacional}

En este trabajo se toma en consideración, especialmente y como han hecho otros autores (Addae, Parboteeah y Velinor, 2008; Rylander, Strutton y Pelton, 1997; Van Vuuren, de Jong y Seydel, 2008), la propuesta de tres componentes del CO de Allen y Meyer (1990): a) el CA refleja la implicación emocional del trabajador con los valores y metas de "su" empresa, el disfrute en la relación laboral y un sentimiento de lealtad y pertenencia a "su" empresa; b) el CN contempla un sentido de deber, obligatoriedad interna y responsabilidad hacia la empresa que resulta de las obligaciones acordadas o tácitas compartidas por las partes, una cierta obligatoriedad interna; c) el CC indica la intención de continuar trabajando en la misma empresa.

Como han sugerido otros investigadores, existe un ciclo de reforzamiento del CO (Rylander et al., 1997). El CA crea unos vínculos emocionales que pueden conducir al trabajador a desarrollar un sentido de responsabilidad y deber con su empresa $(\mathrm{CN})$. Lógicamente la relación inversa no tiene por qué producirse, pues el trabajador puede actuar correctamente porque es su deber $(\mathrm{CN})$ y no sentir afectos hacia la empresa ni estar contento en su trabajo (CA). Por otra parte, los lazos emocionales del CA pueden desembocar en el deseo e intención de continuar con la relación en el futuro (CC) (Rylander et al., 1997). La teoría de la identidad social — que trata de conceptualizar la relación entre el individuo y la organización 
en términos de procesos de identificación social y poniendo énfasis en los grupos sociales que el individuo utiliza como referencia (Ashforth \& Mael, 1989)— sirve de apoyo a la influencia aquí propuesta del CA, que refleja un pensamiento en el colectivo (en "nosotros") más que sólo en el individuo (en el "yo") sobre el CN y el CC. El CN puede conducir al CC, si bien es un efecto distinto del que proviene del CA porque en el primer caso es el sentido de "hacer lo correcto" lo que lleva a continuar la relación, mientras que en el segundo caso es la implicación emocional la que genera la continuidad. Como sugirieron Meyer y Smith (2000), los trabajadores pueden sentir la obligación de continuar trabajando en la misma empresa sin una vinculación afectiva o sentimientos positivos hacia la organización. Así, $H_{l}$ : El CA influye positivamente en el $C N\left(H_{1 a}\right)$ y en el CC $\left(H_{l b}\right)$. El CN influye positivamente en el $\mathrm{CC}\left(\mathrm{H}_{1 \mathrm{l}}\right)$.

\section{Antecedentes del compromiso afectivo}

Uno de los posibles antecedentes del CA del trabajador es la confianza. En el contexto de las relaciones laborales, la confianza del trabajador es la seguridad emocional que lo lleva a pensar que la empresa es responsable y se preocupa por él (adaptación de Mayer et al., 1995, y Singh y Sirdeshmukh, 2000, para el contexto laboral). En otros trabajos ya se ha resaltado la importancia de la confianza del trabajador en sus compañeros, en su jefe, empleador o supervisor (Brashear, Boles, Bellenger y Brooks, 2003; Cunningham y MacGregor, 2000; Matzler y Renzl, 2006; Robinson, 1996) y se ha considerado parte del contenido de la relación laboral entendida como contrato psicológico (Winter y Jackson, 2006). Son muchos los trabajos que, siguiendo el enfoque de marketing relacional y la teoría compromiso-confianza propuesta por Morgan y Hunt (1994), han contemplado la confianza como el principal antecedente del compromiso. Frente a otros trabajos que han propuesto la influencia de la confianza sobre el CO de manera global (Brashear $e t$ al., 2003), aquí se propone su efecto sobre la generación de un CA (Rylander et al., 1997).

Un segundo determinante del CA es la satisfacción laboral, que definimos como los sentimientos positivos derivados de la interacción con sus compañeros y jefes y de todos los aspectos del trabajo, como el sueldo o el horario de trabajo (Brashear et al., 2003). Al igual que la confianza, la satisfacción es una de las variables fundamentales en el enfoque de marketing relacional para lograr retener a los clientes en cualquier organización a través de la creación de lazos afectivos entre las partes, como los implícitos en el CA. En las relaciones laborales, y aunque menos que en 
el caso de la confianza, también se ha enfatizado el papel de la satisfacción como variable estrechamente relacionada con el CO (Addae et al., 2008; Bayona et al., 2000; Brashear et al., 2003; Dunham, Grube y Castenada, 1994).

Como tercer antecedente del CA se consideran las normas relacionales o normas cooperativas que rigen la relación laboral y que pueden dar como resultado una comunicación más personal, cara-a-cara entre la empresa y los trabajadores y, finalmente, una alineación de los intereses de las partes implicadas en la relación laboral (Winter y Jackson, 2006). De las 28 normas relacionales identificadas por Macneil (1980) en su teoría de la contratación relacional, y al igual que en otros trabajos (Gundlach y Achrol, 1993; Jap y Ganesan, 2000; Wathne y Heide, 2000), en este estudio se analizan, desde la percepción del trabajador, las cuatro más importantes en el contexto laboral: flexibilidad (capacidad de adaptación a las circunstancias cambiantes que puedan surgir en la relación laboral), solidaridad (lealtad de las partes ante cualquier diversidad entendiendo que las responsabilidades se asumen conjuntamente), intercambio de información (deseo de compartir voluntariamente información relevante para las partes implicadas en una relación laboral) y participación (intención de las partes de establecer objetivos y tomar decisiones sobre aspectos de la relación laboral de forma conjunta), propuesta adaptada de Macneil (1980) para el contexto laboral.

A diferencia de la confianza y la satisfacción, los investigadores rara vez han estudiado empíricamente las normas relacionales como posible antecedente del CO (sólo nos consta el trabajo de Brashear et al., 2003). La existencia de normas relacionales en la empresa puede conducir a una implicación emocional (CA) del trabajador con la empresa. La teoría del intercambio social (Homans, 1961) enfatiza la importancia de la interacción entre los miembros de un grupo de acuerdo con las normas y cohesión grupal para facilitar los intercambios. Esta teoría puede así apoyar la hipótesis propuesta en este trabajo para el ámbito laboral. En esta línea y en otros trabajos teóricos se ha sugerido que las normas relacionales hacen que las partes se fijen más en intereses comunes que en individuales y refuerzan el CO (Allen y Meyer, 1990; Jap y Ganesan, 2000; Rylander et al., 1997).

El último antecedente propuesto del CA es de naturaleza económica, es decir, el de la percepción de oportunismo, el cual está estrechamente relacionado con la ruptura de la relación laboral. Ésta es una de las variables importantes en la teoría de costos de transacción del enfoque contractual de economía de la empresa y se estudió inicialmente como el incumplimiento activo de un contrato explícito (Wathne 
y Heide, 2000), pero actualmente se reconoce que el oportunismo se puede manifestar de forma activa (o por comisión) o de forma pasiva (o por omisión). En concreto, en el ámbito laboral, el oportunismo es el incumplimiento de los acuerdos explícitos o implícitos, el engaño o mentira, la distorsión de los hechos o la ocultación de información relevante para el trabajador (adaptación de Wathne y Heide, 2000, al contexto laboral). Cuanto mayor sea la percepción de comportamientos empresariales oportunistas (búsqueda del propio interés por encima del interés de todas las partes, ocultación o distorsión de información, engaño o incumplimiento de acuerdos, Ping, 1993) o la violación del contrato psicológico implícito en la relación laboral entre el trabajador y la empresa (Robinson y Rousseau, 1994), más se debilita el vínculo que une a ambas partes y que representa el CA con la empresa. Otros autores ya han propuesto que la ruptura de la relación laboral supone una reducción del CO (Robinson, 1996) o de la intención de permanecer en la misma empresa (Robinson y Rousseau, 1994). Siguiendo el razonamiento anterior, $H_{2}$ : La confianza $\left(H_{2 a}\right)$, la satisfacción $\left(H_{2 b}\right)$ y las normas relacionales $\left(H_{2 c}\right)$ influyen positivamente en el $C A$, mientras que el oportunismo $\left(H_{2 d}\right)$ influye negativamente en el CA.

\section{Antecedentes del compromiso normativo}

El primer determinante del CN que se analiza aquí es la confianza. Aunque tras la revisión de la literatura realizada no se han encontrado trabajos que hayan contrastado explícitamente esta relación, aquí se propone que la confianza en la buena fe de la empresa puede incidir directamente en la generación de un sentido de obligación, deber y responsabilidad en el trabajo $(\mathrm{CN})$, sin tener que generar necesaria y previamente una implicación emocional con la empresa como la que subyace en el CA. Además de la confianza, la percepción de oportunismo empresarial también puede reducir el grado de $\mathrm{CN}$ del trabajador. Así, es probable que ese tipo de comportamientos por parte de la empresa contradiga las expectativas del trabajador sobre lo que debe ser una actuación correcta de la empresa e implique una ruptura de la relación laboral y del contrato psicológico que conlleva. Esta ruptura del contrato psicológico se asocia con una reducción de las obligaciones laborales, de las tareas propias del trabajo, de la intención de permanecer en la empresa, así como de la confianza y cooperación del trabajador con la empresa (Robinson, 1996).

Finalmente, otro posible antecedente del CN es la percepción de dependencia, entendida como el grado en que el trabajador siente que necesita mantener la relación laboral para lograr sus objetivos profesionales y vitales (adaptación de Ganesan, 
1994, al contexto laboral). La dependencia aumenta cuantos más beneficios o ventajas ofrece la relación laboral y cuanto menor sea la disponibilidad de alternativas o los costos de cambio (Kim y Frazier, 1997). El contrato psicológico tradicional (McDonald y Makin, 2000) implica dependencia entre las partes y seguridad laboral a cambio del compromiso del trabajador. La escasez de alternativas laborales y los costos percibidos en caso de abandono de la organización pueden hacer que el trabajador se comprometa con la empresa en la que trabaja (Addae et al., 2008; Allen y Meyer, 1990; Dunham et al., 1994; Meyer y Allen, 1997). El sentimiento por parte del trabajador de dependencia de la empresa puede hacer que aquél se sienta obligado a cumplir bien con su trabajo y ser responsable en el mismo (CN), lo que puede, a su vez, terminar en un compromiso duradero. De acuerdo con las teorías motivacionales, el papel que ejerce la dependencia sobre el CN tiene relación con la teoría X de McGregor (1960) quien supone que el individuo actúa motivado por obligación porque le disgusta el trabajo y no tiene iniciativa, mientras que los antecedentes del CA tienen relación con las políticas implícitas en la teoría Y de McGregor (1960) y la teoría Z de Ouchi (1980), más referidas a la satisfacción y productividad obtenidas con los desafíos del trabajo. Además de estas influencias directas, la satisfacción laboral y las normas relacionales pueden influir indirectamente en el CN a través del CA dado que requieren un mayor tiempo de desarrollo (Jap y Ganesan, 2000; Brown, Dev y Lee, 2000; Cannon, Achrol y Gundlach, 2000); por tanto, primero generarán un clima laboral adecuado y a través de la implicación afectiva del trabajador será cuando influyan en el deber de trabajar correctamente $(\mathrm{CN})$. De esta manera, $H_{3}$ : La confianza $\left(H_{3 a}\right)$ y la percepción de dependencia $\left(H_{3 b}\right)$ influyen positivamente en el $C N$, mientras que la percepción de oportunismo $\left(\mathrm{H}_{3 \mathrm{c}}\right)$ influye negativamente en el $\mathrm{CN}$.

\section{Relaciones entre los antecedentes del compromiso organizacional}

Además de las propias hipótesis correspondientes a los antecedentes de las dimensiones del CO y como señalan Ooi, Bakar, Arumugam, Vellapan y Loke (2007) es importante estudiar los determinantes de la satisfacción laboral. Siguiendo un enfoque relacional, la confianza del trabajador en la empresa y en otros compañeros (Brashear et al., 2003; Cunningham y MacGregor, 2000; Matzler y Renzl, 2006), así como el trabajo en equipo (Ooi et al., 2007) ejercen un gran impacto en su satisfacción y en el CO. Para la relación vendedor-comprador, Terawatanavong, Whitwell y Widing (2007) encuentran que tanto la confianza como las normas cooperativas o relacionales inciden en la satisfacción del comprador en el comienzo y mantenimiento de la relación. De esta forma, es de esperar que el trabajador que 
confía en las buenas intenciones de la empresa en la que trabaja y que percibe que ésta lo deja participar, le proporciona información de su interés y comparten responsabilidades para que esté más satisfecho. Por ello, $\mathrm{H}_{4}$ : La confianza $\left(H_{4 a}\right)$ y las normas relacionales $\left(H_{4 b}\right)$ influyen positivamente en la satisfacción.

Las hipótesis justificadas previamente se reflejan en el siguiente conjunto de ecuaciones que trataremos de contrastar en este trabajo (variable dependiente a la izquierda y variables independientes a la derecha):

$\mathrm{H}_{1:}$ Compromiso de continuidad=compromiso normativo+compromiso afectivo.

$\mathrm{H}_{2}$ : Compromiso normativo $=$ compromiso afectivo.

$\mathrm{H}_{3}$ : Compromiso afectivo=confianza+satisfacción+normas relacionales-percepción de oportunismo.

$\mathrm{H}_{4:}$ Compromiso normativo=confianza+percepción de dependencia-percepción de oportunismo.

$\mathrm{H}_{5:}$ Satisfacción=confianza+normas relacionales.

\section{Metodología}

Para el contraste del modelo propuesto en este trabajo (figura 1) se eligió la relación laboral del trabajador mexicano con la empresa en la que tiene una mayor dedicación laboral, en el caso en que trabaje en más de una. Se contrastó el modelo e hipótesis propuestos a partir de la información recogida a través de encuestas personales — realizadas en septiembre y octubre de 2007- con una muestra aleatoria de trabajadores mexicanos de Monterrey (sin considerar a los empresarios y los trabajadores por cuenta propia o autónomos). El número de cuestionarios válidos fue de 204 de un total de 300 intentos, dado que se eliminaron cuestionarios de personas que actualmente no trabajan y hubo personas que no tenían tiempo para responderlo, por lo que la tasa de respuesta fue del $68 \%$. El error de muestreo fue del $9.7 \%$ para un nivel de confianza del $95 \%$ y el caso más desfavorable. Además, se realizó un estudio piloto mediante entrevistas en profundidad con una muestra de conveniencia de diez trabajadores (cinco hombres y cinco mujeres, tres menores de 30 años, cuatro de entre 31 y 50 años y tres mayores de 50 años) y cinco directivos (tres hombres y dos mujeres, dos menores de 50 años y tres mayores de 50 años) de distintas empresas que ayudaron a comprender la situación interna de la organización y la cultura mexicana, así como a adaptar las medidas al contexto laboral de una forma adecuada a través de la revisión del cuestionario. La muestra engloba 
trabajadores con diferentes características, lo que nos permite tener información de todo tipo de éstos. Así, el $73.73 \%$ son hombres y el $26.27 \%$ son mujeres. En cuanto a la edad quedan representados todos los niveles: de 18 a 25 años (11.8\%), de 25 a 34 años (28.2\%), de 35 a 44 años (39.2\%), de 45 a 54 años (16.6\%) y mayores de 55 años (4.2\%). El sueldo mensual predominante en la muestra es menor de 1275 dólares: menos de 850 dólares (27.6\%), entre 850 y 1274 dólares (24.8\%), entre 1 275 y 1769 dólares (21.8\%), entre 1770 y 2549 dólares (21.8\%) y más de 2549 dólares (4\%). Además, en la muestra hay trabajadores con estudios universitarios (61.39\%) y sin ellos (38.61\%); con hijos (79.2\%) y sin ellos (21.8\%); con contrato fijo/indefinido (63.36\%) y con contrato temporal (36.64\%). Las empresas en las que trabajan los encuestados de la muestra también reúnen variadas características porque pertenecen a distintos sectores (el 22.8\% son del sector servicios), son de distinto tamaño (39.79\% pequeñas, $59.18 \%$ medianas y $1.03 \%$ grandes) y tienen un grado de experiencia en el mercado (63.6\% lleva menos de 10 años, $87.9 \%$ lleva menos de 25 años).

Las variables de este estudio fueron medidas mediante escalas Likert de cinco posiciones que van de 1 (totalmente en desacuerdo o insatisfecho) a 5 (totalmente de acuerdo o satisfecho) (ver Anexo con las preguntas de la encuesta). Los estudios de referencia para medir las variables fueron los siguientes: para el CN, Allen y Meyer (1990) y Gruen, Summers y Acito (2000); para el CA, Allen y Meyer (1990) y Brashear et al. (2003); para el CC, Kim y Frazier (1997); para la confianza y la satisfacción, Brashear et al. (2003); para las normas relacionales, Brashear et al. (2003), Gundlach y Achrol (1993) y Jap y Ganesan (2000); para el oportunismo, Morgan y Hunt (1994) y Ping (1993); para la dependencia, Allen y Meyer (1990) y Ruyter y Wetzels (1999). La extensa literatura revisada y utilizada como referencia asegura la validez de contenido de las escalas de medida.

El procedimiento que se siguió para el contraste de las hipótesis propuestas y los índices utilizados para juzgar la procedencia de eliminar ítemes y para detectar problemas de especificación de los modelos fueron los recomendados por Bagozzi y Yi (1988). De esta forma, se partió de un análisis descriptivo inicial, seguido de un proceso de depuración de las escalas de medición con el fin de que fueran fiables (coeficientes alpha de Cronbach y de confiabilidad compuesta), unidimensionales (análisis factoriales exploratorio y confirmatorio) y válidas de forma convergente y discriminante (análisis factoriales confirmatorios y comprobación de que la varianza extraída es mayor que el cuadrado de la correlación con otros constructos). Posteriormente, para la estimación de las relaciones causales propuestas, se 
recurrió a la modelización de ecuaciones estructurales que tiene grandes ventajas para este tipo de estudios causales, pues es apropiada cuando se estudian variables latentes o/y variables multidimensionales y se quiere estimar un conjunto de ecuaciones simultáneas (las variables pueden ser independientes en unas ecuaciones y dependientes en otras); de la misma forma, proporciona índices de ajuste individuales y globales. Se recomienda aplicar esta metodología a muestras a partir de 200 individuos y que modeliza el error de medida (Jöreskog y Sörbom, 1996; Cepeda y Roldán, 2004; Hair et al., 2001; Bagozzi y Yi, 1988). Para los análisis hemos seguido los linamientos de esos autores y se utilizaron los programas SPSS 15.0 y LISREL 8.7.

\section{Resultados}

El análisis univariante realizado para las variables no reveló ningún dato extraño con capacidad para distorsionar los resultados. Asimismo, se calculó la matriz de correlaciones (cuadro 1) que indica que la media de oportunismo percibido es baja, que las correlaciones de las dimensiones del $\mathrm{CO}$ con los antecedentes relacionales o interpersonales son más altas y con los antecedentes económicos son más bajas. Posteriormente, comenzamos el proceso de validación de las escalas de medición. Así, se realizó una primera prueba de que las escalas utilizadas eran unidimensionales mediante un análisis factorial exploratorio, en concreto, un análisis de ejes principales con rotación varimax para cada grupo de variables consideradas y confirman la existencia de tantos factores como variables y niveles se habían propuesto (cuadro 2): tres para el compromiso (CA, $\mathrm{CN}$ y $\mathrm{CC}$ ), tres para las variables relacionales o interpersonales (la confianza en la empresa, la satisfacción laboral y uno referido a las normas relacionales) y dos para las variables económicas contempladas (la percepción de oportunismo y la de dependencia). Con estas escalas, se realizaron sendos análisis factoriales confirmatorios (método de máxima verosimilitud) con el fin de determinar el grado de validez de cada escala, un concepto más amplio que el de confiabilidad. Con relación al caso del compromiso, variable multidimensional, éste se comprobó mediante un análisis factorial confirmatorio, de ello se concluye que un modelo de tres dimensiones del compromiso es superior al modelo de un factor (Dif. $\chi^{2}(1)=19.663 / \mathrm{p}<.01$ ) y al de dos factores (Dif. $\left.\chi^{2}(1)=22.998 / \mathrm{p}<.01\right)$.

Tras eliminar las variables que presentaban problemas, las salidas para los modelos de medida reespecificados y ajustados se recogen en el cuadro 2, donde se puede observar que los modelos ajustan bien a juzgar por los valores de las me- 
didas de bondad del ajuste. El estadístico Ji-Cuadrado indica un ajuste adecuado para el tamaño de la muestra, al igual que los indicadores GFI (índice de ajuste global), NFI (índice de ajuste normado), CFI (índice de ajuste comparativo), IFI (índice de ajuste incremental) y RMSEA (raíz media residual estandarizada), los que arrojan buenos valores (superiores a 0.90 en GFI, NFI, CFI e IFI y en torno a 0.05 en RMSEA) para aceptar el ajuste de los modelos. Además, se muestran los valores del coeficiente alpha de Cronbach y del índice de confiabilidad compuesta como indicadores de la confiabilidad de cada escala depurada. Como se ve en el cuadro 2, los valores del coeficiente alpha exceden el valor recomendado de 0.7, los del índice de confiabilidad compuesta superan el valor de 0.6 y también los valores de la varianza extraída son mayores de 0.5. Finalmente, se comprobó la validez discriminante de las medidas al corroborar que la correlación al cuadrado de los constructos (ver cuadro 1) es menor que la varianza extraída (ver cuadro 2) (Bagozzi \& Yi, 1988).

Para la estimación del modelo latente global, se realizó un análisis estructural, en concreto un análisis path considerando las escalas depuradas previamente. Una vez analizados los valores de los estimadores y los correspondientes valores del estadístico " $t$ " (figura 2 con los efectos directos y cuadro 3 con los efectos indirectos), se puede observar cómo el modelo ajusta bien y se corroboran la mayor parte de las hipótesis propuestas con un nivel de significación del .05: $\mathrm{H}_{1 \mathrm{a}}, \mathrm{H}_{1 \mathrm{~b}}$, $\mathrm{H}_{1 \mathrm{c}}, \mathrm{H}_{2 \mathrm{~b}}, \mathrm{H}_{2 \mathrm{c}}, \mathrm{H}_{2 \mathrm{~d}}, \mathrm{H}_{3 \mathrm{a}}, \mathrm{H}_{3 \mathrm{~b}}, \mathrm{H}_{4 \mathrm{a}}$ y $\mathrm{H}_{4 \mathrm{~b}}\left(\mathrm{H}_{2 \mathrm{~d}}\right.$ y $\mathrm{H}_{3 \mathrm{~b}}$ con un nivel de significación del .10). Unicamente hay dos hipótesis que no se verifican en este estudio: $\mathrm{H}_{2 \mathrm{a}} \mathrm{y} \mathrm{H}_{3 \mathrm{c}}$. Si valoramos la parsimonia del modelo, observamos que el criterio de información de Akaike (AIC) y el consistente (CAIC) son inferiores en el modelo estimado que en el modelo saturado y en el modelo de variables independientes, lo que indica parsimonia del modelo (Hair et al., 2001).

Además de los efectos directos, el CA media el efecto de ciertos antecedentes interpersonales sobre el $\mathrm{CN}$ porque existe un efecto indirecto significativo de la satisfacción $(\lambda=0.171, \mathrm{t}=2.829)$ y de las normas relacionales $(\lambda=0.191, \mathrm{t}=3.227)$ sobre el CN a través del CA (cuadro 3 ). Asimismo, la confianza, las normas relacionales y la satisfacción ejercen un efecto indirecto significativo sobre el CC. Los resultados muestran que son más importantes los antecedentes relacionales que los económicos para lograr el CA y el CN del trabajador. 


\section{Conclusiones}

En este artículo, se ha propuesto el estudio de las relaciones laborales que entabla el trabajador con la empresa desde un marco teórico que utiliza argumentos de teorías relacionales, contractuales y sociopsicológicas para justificar las hipótesis propuestas. Ese enfoque ha permitido proponer un modelo que distingue antecedentes del CN, CA y CC, de naturaleza relacional o interpersonal y de naturaleza económica. La mayor parte de las hipótesis propuestas son nuevas y no se han encontrado otros trabajos en la literatura de las relaciones laborales que contrasten empíricamente la influencia de variables relacionales-interpersonales y económicas sobre distintas dimensiones del CO. Podemos extraer de este trabajo interesantes implicaciones para el ámbito académico y profesional, los cuales detallamos a continuación.

Los resultados de este estudio, aplicado a un caso mexicano, corroboran las relaciones entre las dimensiones del $\mathrm{CO}$ propuestas inicialmente por Allen y Meyer (1990): el CC viene determinado por el CA y el CN. A diferencia de lo que se ha observado en otros estudios sobre CO (Gruen et al., 2000), la importancia del CA y $\mathrm{CN}$ en la muestra mexicana es similar para lograr que el trabajador permanezca en la empresa. Por un lado, como indica Elahee y Brooks (2004), para los mexicanos son muy importantes los sentimientos en la toma de decisiones y las opiniones están cargadas de un contenido emocional importante, de modo que si existe CA, influirá en su CC. Por otro lado, la cultura colectivista y el nivel de valores y de deber moral en el caso de los individuos mexicanos hace que su CN sea tan importante como el CA (Hofstede, 1980).

De los antecedentes del CA inicialmente propuestos en este trabajo, al seguir el enfoque de marketing relacional, las teorías de la identidad social y del intercambio social y la teoría de la contratación relacional, los que influyen significativamente son la satisfacción laboral y las normas relacionales y, en menor medida, la percepción de oportunismo. Estos resultados confirman que el CA del trabajador está relacionado positivamente con la percepción de apoyo organizacional y con la satisfacción laboral (Allen y Meyer, 1990). Los directivos mexicanos tienden a implicar a los trabajadores mediante su trabajo en equipo y su participación en la toma de decisiones, en la fijación de objetivos y en la realización de sugerencias (Rodríguez, 2005). Las relaciones personales, la personalización y las normas son relevantes para gobernar las relaciones (Elahee y Brooks, 2004). Además, es interesante notar que la generación y mantenimiento de afectos del trabajador hacia 
la empresa (CA) es sensible al oportunismo empresarial, lo que confirma otros estudios que resaltan las consecuencias negativas de la ruptura de la relación laboral entre el trabajador y la empresa (el contrato psicológico según Robinson y Rousseau, 1994). No obstante, no es un gran efecto y es que en México a veces los individuos no perciben ciertos comportamientos como el incumplimiento de promesas o el oportunismo porque su intención principal es tratar de satisfacer a los demás (Elahee y Brooks, 2004). Incluso ante la percepción de oportunismo, muchos trabajadores siguen contribuyendo a la empresa pese a la ruptura de la relación laboral por razones como la percepción de inseguridad en el trabajo o dependencia (Robinson, 1996; Ruiz y Llorens, 2004) o por el clima de satisfacción laboral y normas relacionales que se han desarrollado durante la relación de trabajo y que contribuyen a alinear el contrato psicológico implícito en esa relación laboral (Winter y Jackson, 2006).

De los antecedentes del CN, el principal es el propio CA; otros dos que influyen significativamente son la confianza y la dependencia percibida. En el trabajo aquí realizado, la confianza es un antecedente importante del $\mathrm{CN}$, lo que corrobora la relación causal ya contrastada en numerosos estudios propuesta en la teoría compromiso-confianza de Morgan y Hunt (1994). La dependencia percibida también influye en el CN, aunque ejerce menor efecto que la confianza o el CA, por lo que la vía de la dependencia no parece la más apropiada para lograr que los trabajadores sientan y cumplan con su deber correctamente. El ambiente de negocios, económico y laboral en México es más inestable que en otros países como EEUU o Europa (Elahee y Brooks, 2004), lo cual puede hacer que ciertos trabajadores mexicanos se sientan cómodos dependiendo de la empresa para evitar la incertidumbre y así se sientan responsables en el trabajo $(\mathrm{CN})$. Los resultados también indican que el oportunismo empresarial no reduce el $\mathrm{CN}$ del trabajador. Hay dos posibles explicaciones a este resultado: una que ya hemos comentado referente a que la inseguridad laboral puede desembocar en un CN (Robinson, 1996; Ruiz y Llorens, 2004), pese a la percepción de oportunismo; otra posible es que el CN representa cierta obligatoriedad o necesidad de trabajar y la percepción de oportunismo no tiene por qué minorar la realización de las tareas acordadas en un contrato, aunque sí reduzca el CA.

Además, la confianza y las normas relacionales se revelan como una forma de generar o aumentar la satisfacción laboral del trabajador mexicano para, así, lograr el CA y el CC del trabajador, lo que confirma empíricamente las propuestas de otros autores, más en el caso de la confianza que en el de las normas relacionales 
(Brashear et al., 2003; Matzler y Renzl, 2006; Ooi et al., 2007). La importancia de los antecedentes relacionales es tal que ejercen efectos indirectos significativos en el CN y en el CC del trabajador a través del CA.

Por todo lo anterior y siguiendo los resultados obtenidos con la muestra de trabajadores de Monterrey podemos extraer las siguientes implicaciones profesionales. Las empresas podrían tener dos posibles vías para lograr el CO del trabajador: a) una vía indirecta a través del CA y del CN que consiste en generar un clima de satisfacción y desarrollo de normas relacionales que haga surgir en el trabajador un CA que finalmente le llevará a trabajar de forma responsable $(\mathrm{CN})$ y desear seguir en la empresa (CC); b) una vía más directa independiente del nivel de CA y que consiste en apelar a la percepción de dependencia del trabajador mexicano (por ejemplo, señalando o haciendo que el trabajador sea más consciente de las ventajas adquiridas durante la relación laboral y que perdería en caso de cambio de lugar de trabajo), lo que les hace comprometerse en un sentido normativo y así tener intención de seguir en la misma empresa. Se puede afirmar que la primera vía de compromiso implica una motivación más intrínseca del individuo (Van Vuuren et al., 2008), relacionada con lo que implica un estilo de dirección más participativo según la teoría motivacional tipo Y o Z de McGregor (1960) o la teoría Z de Ouchi (1982). Esta primera opción aporta mayor solidez a la relación laboral y no sólo una mayor duración de esa relación. Por el contrario, la segunda vía conlleva una motivación extrínseca más relacionada con el estilo de mando autoritario propuesto por la teoría motivacional X de McGregor (1960). De acuerdo con Addae et al. (2008), si el CC está basado en la necesidad (como sucede con el CN o con la creación de dependencia) es probable que los trabajadores se limiten a hacer lo mínimo para cumplir su contrato y mantener su trabajo. De hecho hay estudios que indican que, pese a la tendencia al colectivismo en México, hay trabajadores que prefieren no realizar sugerencias ni hablar abiertamente con sus supervisores por temor a asumir riesgos, que prefieren no trabajar en equipo y que el estilo de mando sea autoritario (Elahee y Brooks, 2004). De esta forma se consigue que la relación laboral perdure, pero no es una relación sólida y deseada realmente.

El oportunismo empresarial no parece reducir el nivel de $\mathrm{CN}$ del trabajador, pero sí su CA. No es conveniente que la empresa se comporte de forma oportunista porque si el trabajador lo percibe existe un serio riesgo de ruptura del contrato psicológico entre el trabajador y la empresa, en cuyo caso la empresa podría tratar de recuperar la confianza de dicho trabajador para mantener el nivel del CN. De hecho, la confianza tiene un peso propio para generar $\mathrm{CN}$ y hacer que el trabajador cumpla lo 
acordado con un sentido de deber y responsabilidad porque confía en la empresa en que trabaja. En caso de percepción de oportunismo, la empresa también podría intentar que el clima laboral siga siendo o vuelva a ser el adecuado para desembocar en el CO del trabajador y enfatizar las normas relacionales u ofrecer aspectos que aumenten la satisfacción laboral de éste como el sueldo, las oportunidades de promoción, el horario o el buen ambiente de trabajo. En esta línea, es conveniente que la empresa analice periódicamente el clima laboral en su empresa y el grado del CO de sus trabajadores, así como los factores que pueden aumentar o disminuir la percepción de dependencia, de oportunismo, la confianza, la satisfacción o las normas relacionales con el fin de diseñar estrategias de dirección de recursos humanos enfocadas en cada caso a aumentar el CN, el CA o ambos.

Como limitaciones y líneas de mejora del trabajo, se resaltan las siguientes observaciones: a) El estudio se ha realizado en un ámbito geográfico determinado, con ciertos valores culturales y una determinada situación política, legal y económica, lo que puede afectar a las relaciones laborales y hacer que las conclusiones empíricas obtenidas no se puedan generalizar a otros contextos diferentes (Elahee y Brooks, 2004; Rodríguez, 2005; Madero, 2010). b) El estudio es de corte transversal, por lo que no se puede apreciar el proceso de creación y desarrollo en el tiempo del CO ni separar los distintos momentos temporales en los que hacen su aparición las variables consideradas; de esta forma, sería aconsejable estudiar la situación laboral concreta de una empresa (un análisis de caso) a lo largo del tiempo para conocer la evolución e influencia de las variables del modelo propuesto. c) Hubiera sido deseable un mayor tamaño de la muestra para conseguir una mayor garantía de los resultados. d) Este trabajo refleja el punto de vista del trabajador, por lo que sería interesante recoger información de otras partes también implicadas en la relación laboral - la organización, supervisores o gerentes - y averiguar si las percepciones son diferentes a las del trabajador (Robinson, 1996). e) Sería recomendable estudiar las diferencias en el desarrollo del CO según el tipo de contrato o el número de años que lleva el trabajador en la empresa, lo que puede implicar, por ejemplo, inseguridad en los trabajadores eventuales (Ruiz y Llorens, 2004); así, sería relevante analizar el efecto moderador del grado de experiencia laboral del trabajador mediante un análisis de ecuaciones estructurales multigrupo. 


\section{Referencias}

Addae, H. M., K. P. Parboteeah y N. Velinor (2008). Role stressors and organizational commitment: public sector employment in St Lucia. International Journal of Manpower 29 (6): 567-582.

Allen, N. J. y J. P. Meyer (1990). The measurement and antecedents of affective, continuance and normative commitment the organization. Journal Occupational Psychology 63: 1-18.

Ashforth, B. E. y F. Mael (1989). Social identity theory and the organization. Academy of Management Review 14 (1): 20-39.

Bagozzi, R. P. y Y. Yi (1988). On the evaluation of structural equation models. Journal of the Academy of Marketing Science 16 (1): 74-94.

Bayona, C., S. Goñi y C. Madorran (2000). Compromiso organizacional: implicaciones para la gestión estratégica de los Recursos Humanos. Revista Europea de Dirección y Economía de la Empresa 9 (1): 139-149.

Brashear, T. G., J. S. Boles, D. N. Bellenger y C. M. Brooks (2003). An empirical test of trust-building processes and outcomes in sales manager-salesperson relationships. Journal of the Academy of Marketing Science 31 (2): 189200.

Brown, J. R., C. S. Dev y D-J. Lee (2000). Managing marketing channel opportunism: the efficacy of alternative governance mechanisms. Journal of Marketing, abril, 64: 51-65.

Cannon, J. P., R. S. Achrol y G. T. Gundlach (2000). Contracts, norms, and plural form governance. Journal of the Academy of Marketing Science 28 (2): 180194.

Cepeda, G. y J. Roldán (2004). Aplicando en la práctica la Técnica PLS en la Administración de Empresas. Actas del XIV Congreso de la Asociación Científica de Economía y Dirección de Empresas (ACEDE). Murcia, España, septiembre, 1-30. 
Cunningham, J. B. y J. MacGregor (2000). Trust and the design of work: complementary constructs in satisfaction and performance. Human Relations 53 (12): $1575-1591$.

Dunham, R. B., J. A. Grube y M. B. Castenada (1994). Organizational commitment: the utility of an integrative definition. Journal of Applied Psychology 79: 370-380.

Elahee, M. y C. M. Brooks (2004). Trust and negotiation tactics: perceptions about business-to-business negotiations in Mexico. Journal of Business \& Industrial Marketing 19 (6): 397-404.

Ganesan, S. (1994). Determinants of long-term orientation in buyer-seller relationships. Journal of Marketing, abril, 58: 1-19.

Guest, D. (1998). Is the psychological contract worth taking seriously? Journal of Organizational Behavior 19: 649-664.

Gruen, T. W., J. O. Summers y F. Acito (2000). Relationship marketing activities, commitment, and membership behaviors in professional associations. Journal of Marketing 64: 34-49.

Gundlach, G. T. y R. S. Achrol (1993). Governance in exchange: contract law and its alternatives. Journal of Public Policy and Marketing 12 (2): 141-155.

Hair, J. F. et al. (2001). Análisis multivariante de datos. $5^{\text {a }}$ ed., Madrid: PearsonPrentice Hall.

Homans, G. (1961). Social Behavior: Elementary Forms. New York: Harcourt, Brace \& World.

Hofstede, G. (1980). Culture’s consequences. Beverly Hills: Sage.

Jap, S. D. y S. Ganesan (2000). Control mechanisms and the relationship life cycle: implications for safeguarding specific investments and developing commitment. Journal of Marketing Research 37: 227-245. 
Jöreskog, K. G. y D. Sörbom (1996). LISREL VIII: Structural equation modeling with the SIMPLIS command language. Chicago: Scientific Software International, INC.

Kim, K. y G. L. Frazier (1997). On distributor commitment in industrial channels of distribution: a multicomponent approach. Psychology and Marketing 14: 847-877.

Macneil, I. R. (1980). The new social contract: an inquiry into modern contractual relations. New Haven: Yale University Press.

Madero, S. M. (2010). Factores relevantes del desarrollo profesional y de compensaciones en la carrera laboral del trabajador. Contaduría y Administración, septiembre-diciembre, 232: 109-130.

Matzler, K. y B. Renzl (2006). The Relationship between Interpersonal Trust, Employee Satisfaction, and Employee Loyalty. Total Quality Management \& Business Excellence 17 (10): 1261-1271.

Mayer, R. C., J. H. Davis y F. D. Schoorman (1995). An integrative model of organizational trust. Academy of Management Review 20 (3): 709-734.

McDonald, D. J. y P. J. Makin (2000). The psychological contract, organisational commitment and job satisfaction of emporary staff. Leadership \& Organization 21 (2): 84-91.

McGregor, D. (1960). El lado humano de las organizaciones. Nueva York: Mc Graw Hill.

Meyer, J. P. y N. J. Allen (1997). Commitment in the workplace: theory, research, and application. Thousand Oaks: Sage.

y C. A. Smith (2000). HRM practices and organizational commitment: test of a mediation model. Canadian Journal of Administrative Sciences 17 (4): 319-331.

Morgan, R. y S. Hunt (1994). The commitment-trust theory of relationship marketing. Journal of Marketing 58: 20-38. 
Ooi, K. B., N. A. Bakar, V. Arumugam, L. Vellapan y A. K. Y. Loke (2007). Does TQM influence employees' job satisfaction? An empirical case analysis. International Journal of Quality \& Reliability Management 24 (1): 62-77.

Ouchi, W. G. (1982). Theory Z. New York: Avon Books.

Ping, R. A. Jr. (1993). The effects of satisfaction and structural constraints on retailer exiting, voice, loyalty, oportunism and neglect. Journal of Retailing 69 (3): 320-352.

Robinson, S. L. (1996). Trust and breach of the psychological contract. Administrative Science Quarterly 41: 574-599.

y D. M. Rousseau (1994). Violating the psychological contract: not the exception but the norm. Journal of Organizational Behavior 15: 245259.

Rodríguez, C. M. (2005). Emergence of a third culture: shared leadership in international strategic alliances. International Marketing Review 22 (1): 67-95.

Ruiz, A. y F. J. Llorens (2004). Efectos de las relaciones laborales en el comportamiento organizacional y en las percepciones de prestación del servicio. Revista Europea de Dirección y Economía de la Empresa 13: 115-130.

Ruyter, K. D. y M. Wetzels (1999). Commitment in auditor-client relationships: antecedents and consequences. Accounting, Organisations and Society 24: $57-75$.

Rylander, D., D. Strutton y L. E. Pelton (1997). Toward a synthesized framework of relational commitment: implications for marketing channel theory and practice. Journal of Marketing Theory and Practice 5 (2): 58-71.

Terawatanavong, C., G. J. Whitwell y R. E. Widing (2007). Buyer satisfaction with relational exchange across the relationship lifecycle. European Journal of Marketing 41 (7/8): 915-938. 
Van Vuuren, M., M. D. T. de Jong y E. R. Seydel (2008). Contributions of self and organisational efficacy expectations to commitment: A fourfold typology. Employee Relations 30 (2): 142-155.

Wathne, K. H. y J. B. Heide (2000). Opportunism in interfirm relationships: forms, outcomes, and solutions. Journal of Marketing 64: 36-51.

Winter, R. y B. Jackson (2006). State of the psychological contract: manager and employee perspectives within an Australian credit union. Employee Relations 28 (5): 421-434. (A) 
Cuadro 1

Descriptivos y matriz de correlaciones de las variables objeto de estudio

\begin{tabular}{|c|c|c|c|c|c|c|c|c|c|c|}
\hline & Media & $\begin{array}{c}\text { Desviación } \\
\text { estándar }\end{array}$ & $\mathbf{C N}$ & CA & $\mathrm{CC}$ & Satisfacción & $\begin{array}{c}\text { Normas } \\
\text { relacionales }\end{array}$ & Confianza & Oportunismo & Dependencia \\
\hline $\mathrm{CN}$ & 3.992 & .761 & - & & & & & & & \\
\hline $\mathrm{CA}$ & 3.886 & .804 & $.638 * *$ & - & & & & & & \\
\hline $\mathrm{CC}$ & 4.035 & .765 & $.658 * *$ & $.637 * *$ & 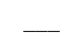 & & & & & \\
\hline Satisfacción & 4.747 & .963 & $.699 * *$ & $.742 * *$ & $.650 * *$ & - & & & & \\
\hline $\begin{array}{c}\text { Normas } \\
\text { relacionales }\end{array}$ & 3.774 & .823 & $.593 * *$ & $.716^{* *}$ & $.606 * *$ & $.783 * *$ & - & & & \\
\hline Confianza & 3.901 & .821 & $.691 * *$ & $.624 * *$ & $.659 * *$ & $.789 * *$ & $.720 * *$ & - & & \\
\hline Oportunismo & 1.343 & 1.029 & -.074 & $-.279 * *$ & $-.248 *$ & -.188 & $-.269 * *$ & -.149 & - & \\
\hline Dependencia & 3.893 & .902 & $.245^{*}$ & .132 & .111 & .148 & $.221 *$ & .194 & -.059 & $\ldots$ \\
\hline
\end{tabular}

** La correlación es significativa al nivel de .01 (bilateral).

* La correlación es significativa al nivel de .05 (bilateral).

$\mathrm{CN}=$ compromiso normativo; $\mathrm{CA}=$ compromiso afectivo; $\mathrm{CC}=$ compromiso de continuidad. 


\section{Cuadro 2}

\section{Resultados de los análisis factoriales exploratorios y confirmatorios de las variables estudiadas}

\begin{tabular}{|c|c|c|c|c|c|c|c|c|}
\hline \multirow{2}{*}{$\begin{array}{l}\text { Variables- } \\
\text { factores } \\
\text { resultantes } \\
\text { del análisis }\end{array}$} & \multirow[t]{2}{*}{$\begin{array}{c}\text { Variables } \\
\text { que componen } \\
\text { el factor }\end{array}$} & \multirow[t]{2}{*}{$\begin{array}{r}\text { Varianza } \\
\text { explicada }\end{array}$} & \multicolumn{2}{|c|}{$\begin{array}{c}\text { Coeficientes } \\
\text { Lambda }\end{array}$} & \multirow{2}{*}{$\mathbf{R}^{2}$} & \multirow{2}{*}{$\begin{array}{c}\alpha \text { Cronbach/ } \\
\text { Confiabilidad } \\
\text { compuesta }\end{array}$} & \multirow{2}{*}{$\begin{array}{l}\text { Varianza } \\
\text { extraída }\end{array}$} & \multirow{2}{*}{$\begin{array}{c}\text { Bondad } \\
\text { del } \\
\text { ajuste }\end{array}$} \\
\hline & & & Lambda & $\mathbf{T}$ & & & & \\
\hline \multirow{4}{*}{$\begin{array}{c}\text { Compromiso } \\
\text { normativo }\end{array}$} & V1 & & .803 & 9.673 & 0.621 & \multirow{4}{*}{$\begin{array}{c}0.7737 / \\
0.9015\end{array}$} & \multirow{4}{*}{0.5677} & \\
\hline & V2 & & .596 & 6.308 & 0.348 & & & \\
\hline & V3 & 46.592 & .726 & -- & 0.514 & & & \\
\hline & V4 & & .693 & 7.753 & 0.467 & & & $\chi^{2}=52.573$ \\
\hline \multirow{5}{*}{$\begin{array}{l}\text { Compromiso } \\
\text { afectivo }\end{array}$} & V5 & & .823 & 9.727 & 0.638 & \multirow{5}{*}{$\begin{array}{l}0.7389 / \\
0.8416\end{array}$} & \multirow{5}{*}{0.564} & GFI $=0.912$ \\
\hline & & & & & & & & $\mathrm{NFI}=0.90$ \\
\hline & V7 & & .749 & -- & 0.485 & & & $\mathrm{CFI}=0.953$ \\
\hline & V8 & 8.698 & .704 & 7.754 & 0.474 & & & $\begin{array}{c}\mathrm{IFI}=0.955 \\
\text { RMSEA }= \\
0.069\end{array}$ \\
\hline & V9 & & .548 & 5.603 & 0.393 & & & \\
\hline \multirow{3}{*}{$\begin{array}{c}\text { Compromiso } \\
\text { de } \\
\text { continuidad }\end{array}$} & V10 & & .831 & -- & 0.655 & \multirow{2}{*}{$\begin{array}{l}0.7756 / \\
0.9464\end{array}$} & \multirow[b]{2}{*}{0.887} & \\
\hline & V12 & 7.486 & .825 & 10.016 & 0.650 & & & \\
\hline & V13 & & .667 & 7.766 & 0.448 & \multirow{5}{*}{$\begin{array}{l}0.8180 / \\
0.9479\end{array}$} & \multirow{5}{*}{0.6827} & \\
\hline \multirow{4}{*}{ Confianza } & V14 & & .666 & 7.739 & 0.446 & & & \\
\hline & V15 & 5080 & .704 & 8.438 & 0.498 & & & \\
\hline & V16 & 5.000 & 699 & 8.346 & 0.492 & & & \\
\hline & V17 & & .789 & -- & 0.545 & & & \\
\hline \multirow{5}{*}{ Satisfacción } & V19 & & .590 & -- & 0.427 & \multirow{5}{*}{$\begin{array}{l}0.8658 / \\
0.9349\end{array}$} & \multirow{5}{*}{0.6282} & \\
\hline & V20 & & .682 & 8.318 & 0.475 & & & $\chi^{2}=102.079$ \\
\hline & $\mathrm{V} 21$ & & .733 & 9.401 & 0.550 & & & $(\mathrm{p}=0.298)$ \\
\hline & V22 & 47.736 & .687 & 8.415 & 0.482 & & & GFI $=0.901$ \\
\hline & $\mathrm{V} 23$ & & .603 & 6.905 & 0.370 & & & $\mathrm{CFI}=0.989$ \\
\hline \multirow{5}{*}{$\begin{array}{c}\text { Normas } \\
\text { relacionales }\end{array}$} & V26 & & .644 & 7.583 & 0.422 & \multirow{5}{*}{$\begin{array}{l}0.8635 / \\
0.9303\end{array}$} & \multirow{5}{*}{0.641} & $\mathrm{IFI}=0.989$ \\
\hline & V27 & & .763 & -- & 0.588 & & & $\begin{array}{c}\text { RMSEA= } \\
0.0256\end{array}$ \\
\hline & V28 & 6.635 & .657 & 7.914 & 0.439 & & & \\
\hline & V29 & & .656 & 7.808 & 0.438 & & & \\
\hline & V30 & & .763 & 8.251 & 0.599 & & & \\
\hline
\end{tabular}




\begin{tabular}{|c|c|c|c|c|c|c|c|c|}
\hline \multirow{2}{*}{$\begin{array}{l}\text { Variables- } \\
\text { factores } \\
\text { resultantes } \\
\text { del análisis }\end{array}$} & \multirow[t]{2}{*}{$\begin{array}{c}\text { Variables } \\
\text { que componen } \\
\text { el factor }\end{array}$} & \multirow[t]{2}{*}{$\begin{array}{r}\text { Varianza } \\
\text { explicada }\end{array}$} & \multicolumn{2}{|c|}{$\begin{array}{c}\text { Coeficientes } \\
\text { Lambda }\end{array}$} & \multirow{2}{*}{$\mathbf{R}^{2}$} & \multirow{2}{*}{$\begin{array}{l}\alpha \text { Cronbach/ } \\
\text { Confiabilidad } \\
\text { compuesta }\end{array}$} & \multirow{2}{*}{$\begin{array}{l}\text { Varianza } \\
\text { extraída }\end{array}$} & \multirow{2}{*}{$\begin{array}{c}\text { Bondad } \\
\text { del } \\
\text { ajuste }\end{array}$} \\
\hline & & & Lambda & $\mathbf{T}$ & & & & \\
\hline \multirow{4}{*}{ Dependencia } & V32 & \multirow{4}{*}{38.132} & .867 & 11.972 & 0.751 & \multirow{4}{*}{$\begin{array}{l}0.8565 / \\
0.9126\end{array}$} & \multirow{4}{*}{0.578} & \\
\hline & V33 & & .748 & 9.313 & 0.560 & & & \\
\hline & V34 & & .882 & -- & 0.777 & & & $\chi^{2}=59.432$ \\
\hline & V36 & & .680 & 8.001 & 0.473 & & & $(\mathrm{p}=0.0050)$ \\
\hline \multirow{6}{*}{$\begin{array}{l}\text { Percepción de } \\
\text { oportunismo }\end{array}$} & V40 & \multirow{6}{*}{29.452} & 0.819 & 12.538 & 0.671 & \multirow{6}{*}{$\begin{array}{l}0.9489 / \\
0.9429\end{array}$} & \multirow{6}{*}{0.6377} & GFI $=0.905$ \\
\hline & V41 & & 0.841 & 13.421 & 0.708 & & & $\mathrm{NFI}=0.932$ \\
\hline & V42 & & 0.892 & 15.946 & 0.796 & & & $\mathrm{CFI}=0.963$ \\
\hline & V43 & & 0.880 & 15.262 & 0.774 & & & $\mathrm{IFI}=0.964$ \\
\hline & V44 & & 0.920 & 17.764 & 0.846 & & & RMSEA = 0.0571 \\
\hline & V45 & & 0.934 & -- & 0.873 & & & \\
\hline
\end{tabular}

Nota. Las variables que no aparecen fueron eliminadas en el proceso de depuración de las escalas mediante los análisis factoriales confirmatorios.

GFI= Índice de ajuste global; NFI= Índice de ajuste normado; CFI= Índice de ajuste comparativo; IFI=Índice de ajuste incremental; RMSEA=raíz media residual estandarizada.

\section{Cuadro 3}

Efectos indirectos resultantes de la estimación del modelo global mediante ecuaciones estructurales

\begin{tabular}{cccc}
\hline & \multicolumn{3}{c}{ Efectos indirectos } \\
\cline { 2 - 4 } & $\mathbf{C N}$ & $\mathbf{C A}$ & $\mathbf{C C}$ \\
\hline Confianza & $.087(2.059)^{* *}$ & $.217(3.244)^{* *}$ & $.316(4.334)^{* *}$ \\
Satisfacción & $.171(2.829)^{* *}$ & --- & $.243(3.274)^{* *}$ \\
Normas & $.191(3.227)^{* *}$ & $.207(3.198)^{* *}$ & $.271(3.940)^{* *}$ \\
Oportunismo & $-.039(-1.480)$ & --- & $-.011(-0.237)$ \\
Dependencia & --- & --- & $.049(1.570)$ \\
CA & --- & --- & $.156(3.079)^{* *}$ \\
CN & --- & --- & -- \\
\hline
\end{tabular}




\section{Figura 1}

\section{Modelo propuesto con variables latentes y observables}

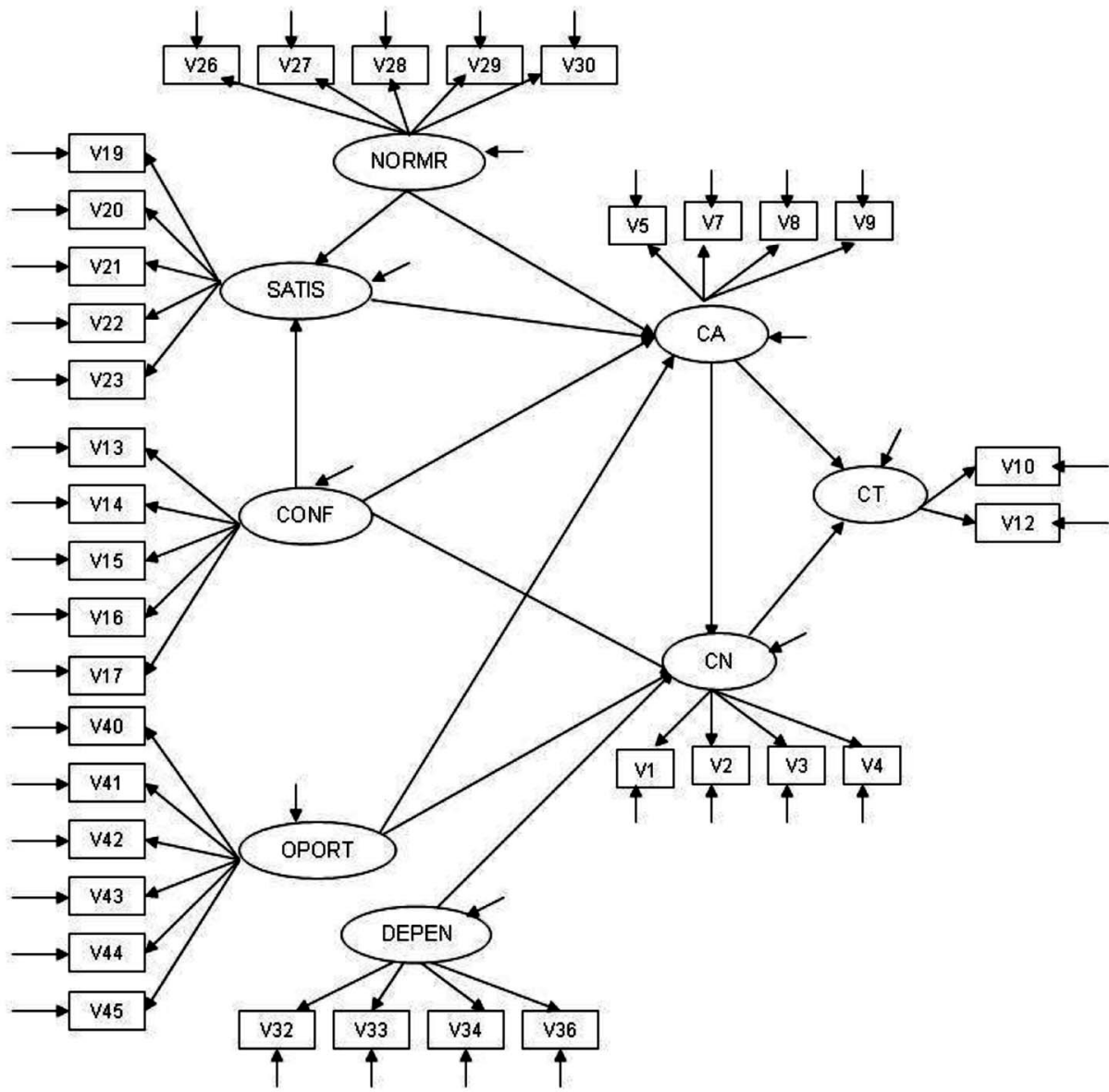

Nota. $\mathrm{CA}=$ compromiso afectivo; $\mathrm{CN}=$ compromiso normativo; $\mathrm{CC}=$ compromiso de continui-

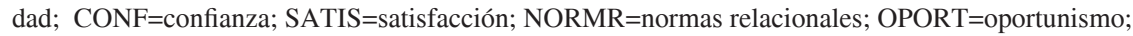
DEPEN=dependencia. 
Figura 2

Modelo propuesto con variables latentes y observables

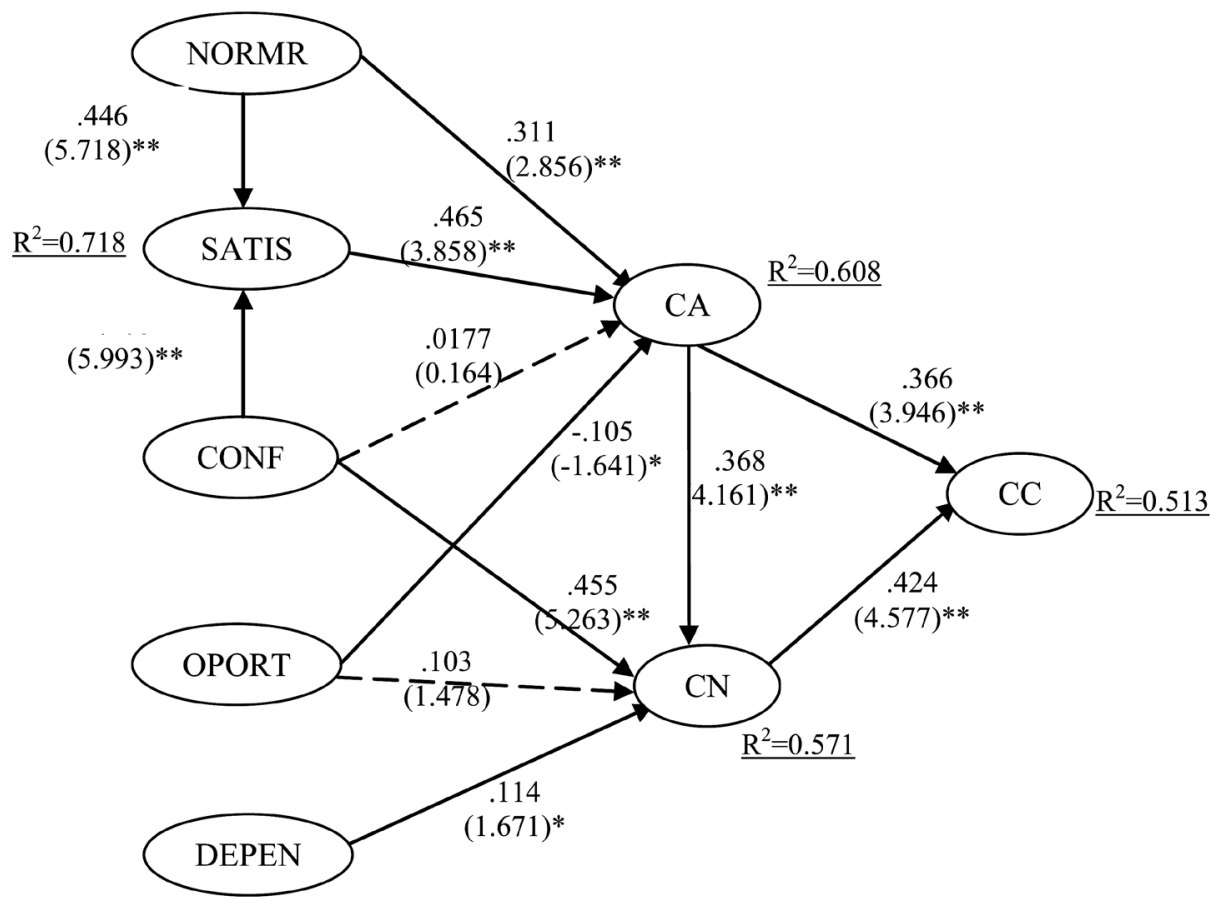

Índices de ajuste global

$\chi^{2}=18.110(\mathrm{p}=0.05) ; \mathrm{GFI}=0.959 ; \mathrm{CFI}=0.989 ; \mathrm{IFI}=0.989 ; \mathrm{NFI}=0.976 ; \mathrm{RMSEA}=0.055$

AIC modelo estimado $=68.928$, independiente $=763.049$, saturado $=72.000$

CAIC modelo estimado $=162.921$, independiente $=791.970$, saturado $=202.144$

Nota. GFI= Índice de ajuste global; NFI= Índice de ajuste normado; CFI= Índice de ajuste comparativo; IFI=Índice de ajuste incremental; RMSEA=raíz media residual estandarizada; $\mathrm{AIC}=$ criterio de información de Akaike; $\mathrm{CAIC}=$ criterio de información de Akaike consistente.

Nota. En líneas continuas aparecen las relaciones propuestas y sí corroboradas en este trabajo. ** Correlación significativa al nivel de .05 (bilateral) /* Significativa al nivel de .10 .

En líneas discontinuas aparecen las relaciones propuestas y no corroboradas en este trabajo. 


\section{Anexo \\ Preguntas de la encuesta utilizada}

\section{Pregunta filtro:}

¿Trabaja en más de una empresa? a) Sí b) NO

Si trabaja en más de una empresa, se pidió al encuestado que refiriera la encuesta sólo a la empresa para la que más horas trabaja.

\section{Preguntas sobre compromiso organizacional.}

(1=totalmente en desacuerdo/5=totalmente de acuerdo)

Es mi deber cumplir las obligaciones de trabajo acordadas con esta empresa ............... $122 \quad 3 \quad 4 \quad 4 \quad 5$

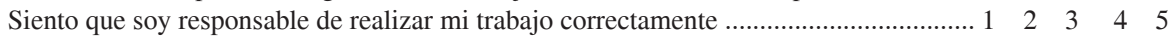

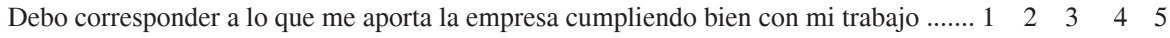

Al menos por el bien de los clientes y la sociedad, debo cumplir mis obligaciones

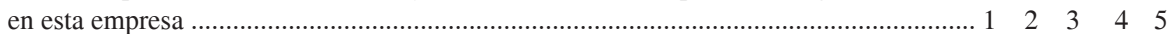

Valoro positivamente mi relación con esta empresa .................................................. $1 \quad 2 \quad 3 \quad 4 \quad 5$

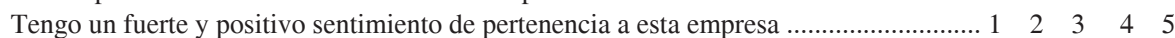

Siento los problemas de la empresa como si fueran míos ........................................... 1 2 $23 \quad 3 \quad 4 \quad 5$

Hablo bien de mi empresa a mis conocidos y amigos.................................................. 1 2 $23 \quad 4 \quad 5$

Mantengo una relación cordial con mis compañeros de la empresa................................. 1 2 $23^{3} 4 \quad 5$

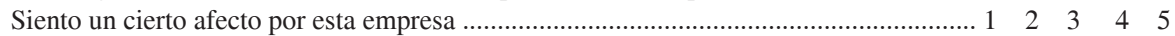

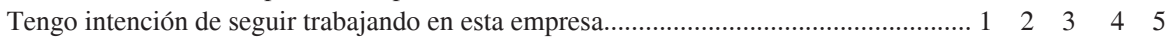

Espero que mi relación con esta empresa dure mucho tiempo....................................... 1 2 2 3 $4 \quad 5$

Me gustaría que la relación que mantengo con esta empresa siga siendo así o incluso

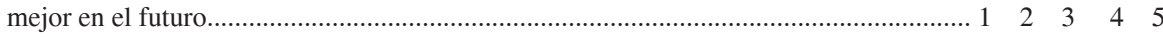

\section{Preguntas sobre satisfacción laboral.}

Sus expectativas respecto a esta empresa (marque con un círculo el número más adecuado):

No se han cumplido en absoluto $\quad \begin{array}{lllllll}1 & 2 & 3 & 4 & 5 & \text { Se han cumplido }\end{array}$ con creces

Cuando pienso en mi relación con la empresa, en general estoy: $\begin{array}{lllll}1 & 2 & 3 & 4 & 5\end{array}$

Con las oportunidades de promoción en la empresa, estoy:

Con el sueldo, estoy:

Con el ambiente de trabajo (relaciones con compañeros y jefes), estoy:...........

Con el trabajo que tengo que realizar, estoy: 


\section{Preguntas sobre normas relacionales:}

(1=totalmente en desacuerdo/5=totalmente de acuerdo)

No me importa trabajar más que otros para conseguir los objetivos comunes ……...................1 $2 \begin{array}{llll}2 & 3 & 4 & 5\end{array}$

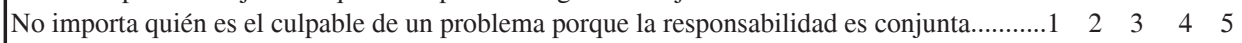

La empresa nos mantiene informados sobre los hechos o cambios que nos pueden afectar.......1 $\begin{array}{lllll}2 & 3 & 4 & 5\end{array}$

La empresa recoge regularmente opiniones y sugerencias de los trabajadores...........................1 $2 \begin{array}{lllll}2 & 3 & 4 & 5\end{array}$

La empresa recibe con agrado las ideas de los trabajadores....................................................... $12 \begin{array}{llll}2 & 3 & 4 & 5\end{array}$

En esta empresa, existe un compromiso de mejora que beneficie a todos y no a alguien

en particular.......

\section{Preguntas sobre confianza y oportunismo:}

\begin{tabular}{|c|c|c|c|c|}
\hline \multirow{2}{*}{\multicolumn{5}{|c|}{ (1=totalmente en desacuerdo $/ 5=$ totalmente de acuerdo $)$}} \\
\hline & & & & \\
\hline \multicolumn{5}{|l|}{ Esta empresa cuenta con excelentes recursos para realizar su actividad } \\
\hline 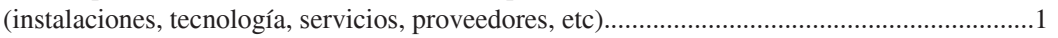 & 2 & 3 & 4 & 5 \\
\hline Creo que el personal está altamente capacitado ............... & 2 & 3 & 4 & 5 \\
\hline intenciones de esta empresa.. & 2 & 3 & 4 & 5 \\
\hline Creo que en esta empresa cumplen lo prometido.... & 2 & 3 & 4 & 5 \\
\hline Considero que me puedo fiar de esta empresa ........ & 2 & 3 & 4 & 5 \\
\hline 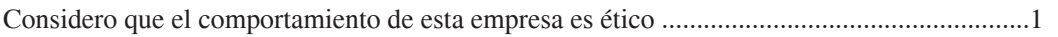 & 2 & 3 & 4 & 5 \\
\hline \multicolumn{5}{|l|}{ Percibo que existe una preocupación especial por conseguir que los trabajadores } \\
\hline & 2 & 3 & 4 & 5 \\
\hline Me gusta el estilo de trabajo de la empresa. & 2 & 3 & 4 & 5 \\
\hline 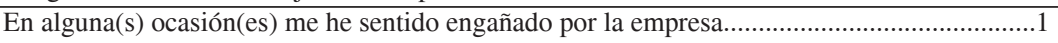 & 2 & 3 & 4 & 5 \\
\hline 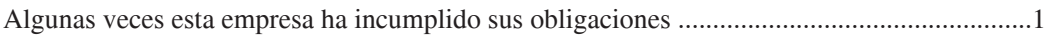 & 2 & 3 & 4 & 5 \\
\hline La empresa trata de eludir sus responsabilidades si pasa desapercibido .................................. & 2 & 3 & 4 & 5 \\
\hline \multicolumn{5}{|l|}{ Percibo que la empresa busca principalmente su propio interés, aunque perjudique a } \\
\hline & 2 & 3 & 4 & 5 \\
\hline Necesito estar pendiente de la empresa para que cumpla lo prometido .. & 2 & 3 & 4 & 5 \\
\hline Esta empresa altera a veces los hechos u oculta la verdad a los trabajadores .. & 2 & 3 & 4 & 5 \\
\hline
\end{tabular}

\section{Preguntas sobre dependencia:}

Considero que dependo de esta empresa.......

(1=totalmente en desacuerdo/5=totalmente de acuerdo)

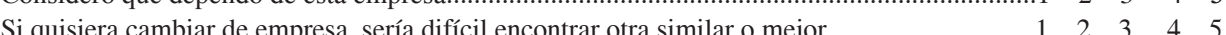

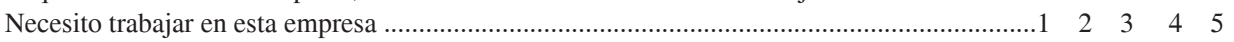

Para cambiar de empresa, sería necesario mucho esfuerzo (monetario, emocional,

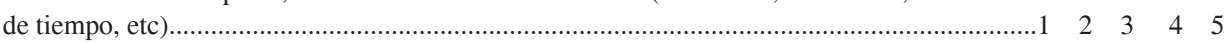

Si cambiase de empresa, perdería las ventajas que he adquirido en ésta.............................. 1 2 $34 \quad 3 \quad 5$

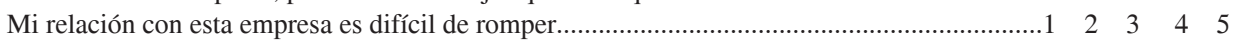


Sexo

\begin{tabular}{|l|l|}
\hline & Hombre \\
\hline & Mujer \\
\hline
\end{tabular}

Edad:

\begin{tabular}{|l|l|}
\hline & De 18 a 24 años \\
\hline & De 25 a 34 años \\
\hline & De 35 a 44 años \\
\hline & De 45 a 54 años \\
\hline & Más de 54 años \\
\hline
\end{tabular}

Nivel de estudios:

\begin{tabular}{|l|l|}
\hline & Sin estudios \\
\hline & Estudios primarios \\
\hline & Estudios medios \\
\hline $\begin{array}{l}\text { Estudios universitarios (ciclo } \\
\text { corto) }\end{array}$ \\
\hline $\begin{array}{l}\text { Estudios universitarios } \\
\text { (licenciatura) }\end{array}$ \\
\hline & Cursos de posgrado/doctorado \\
\hline
\end{tabular}

Tiene hijos: a) sí b) no

Tipo de contrato: a) Temporal b) Indefinido/fijo
Sueldo neto al mes:

\begin{tabular}{|l|l|}
\hline & Menos de 850 dólares \\
\hline & 850-1274 dólares \\
\hline & 1275-1769 dólares \\
\hline & 1770-2549 dólares \\
\hline & Más de 2549 dólares \\
\hline
\end{tabular}

Aspectos de la empresa en que trabaja:

Número de años aproximado que la empresa lleva operando en el mercado: años

Sector en el que opera la empresa:

\begin{tabular}{|l|l|}
\hline & Consumo \\
\hline & Industrial \\
\hline & Servicios \\
\hline
\end{tabular}

Tamaño de la empresa:

\begin{tabular}{|l|l|}
\hline & Pequeña \\
\hline & Mediana \\
\hline & Grande \\
\hline
\end{tabular}

